\title{
The role of meat in the diets of Irish adults (18-90 years)
} aim of this analysis was to estimate the intakes of meat and meat products and their contribution to nutrient intakes in Irish adults. Analyses were based on data from the National Adult Nutrition Survey (2008-2010) (www.iuna.net). A 4-day semi-weighed food diary was used to collect dietary intake data from a nationally representative sample of Irish adults aged $18-90$ years $(\mathrm{n}=1500$ ). Nutrient intake data were analysed using WISP ${ }^{\odot}$ v3.0 based on UK ${ }^{(2)}$ and $\operatorname{Irish}^{(3)}$ food composition tables. For the purpose of these analyses, red meat is defined as unprocessed beef, veal, lamb, pork and venison (including minced or frozen meat). Processed meat is defined as red meat that has been treated with preservatives, excluding salt, but including some cured meat products. Meat intakes from meat products and composite dishes were estimated following disaggregation of the non-meat components (e.g. potatoes, pasta, vegetables, sauces, oils, coatings). Nutrient intakes from meat products and composite dishes (as consumed) were estimated including the non-meat components. Statistical analysis was conducted using SPSS ${ }^{\odot}$ v24.

\begin{tabular}{|c|c|c|c|c|c|c|c|c|c|c|}
\hline & \multicolumn{2}{|c|}{$\begin{array}{c}\text { Total } \\
(n=1500)\end{array}$} & \multicolumn{2}{|c|}{$\frac{18-35 y}{(n=531)}$} & \multicolumn{2}{|c|}{$\begin{array}{c}36-50 y \\
(n=437)\end{array}$} & \multicolumn{2}{|c|}{$\begin{array}{c}51-64 y \\
(n=306)\end{array}$} & \multicolumn{2}{|c|}{$\begin{array}{c}65-90 y \\
(n=226)\end{array}$} \\
\hline & $\mathrm{M}^{*}$ & $\mathrm{~W}^{*}$ & $\mathrm{M}$ & $\mathrm{W}$ & $\mathrm{M}$ & $\mathrm{W}$ & M & $\mathrm{W}$ & M & $\mathrm{W}$ \\
\hline Total meat (g) & 163 & 101 & 175 & 100 & 169 & $98 \cdot 2$ & 151 & 108 & 133 & 101 \\
\hline Red meat (g) & $65 \cdot 9$ & $38 \cdot 2$ & $66 \cdot 0$ & $31 \cdot 3$ & $69 \cdot 5$ & $36 \cdot 5$ & $64 \cdot 4$ & $46 \cdot 8$ & $61 \cdot 0$ & $45 \cdot 3$ \\
\hline Processed meat (g) & $42 \cdot 7$ & $23 \cdot 9$ & 41.9 & $23 \cdot 8$ & $46 \cdot 3$ & 23.5 & $41 \cdot 9$ & $24 \cdot 9$ & $39 \cdot 1$ & $23 \cdot 5$ \\
\hline Poultry and game (g) & $53 \cdot 9$ & $39 \cdot 0$ & $67 \cdot 5$ & 44.9 & $53 \cdot 6$ & 38.2 & $44 \cdot 7$ & $36 \cdot 1$ & $32 \cdot 5$ & 31.8 \\
\hline
\end{tabular}

Contribution (\%) of meat and meat products to total energy and selected nutrient intakes

\begin{tabular}{|c|c|c|c|c|c|c|c|c|c|}
\hline Energy & 19 & 16 & 19 & 16 & 20 & 16 & 18 & 17 & 18 \\
\hline Protein & 43 & 38 & 44 & 39 & 44 & 38 & 42 & 39 & 39 \\
\hline Total fat & 26 & 21 & 26 & 21 & 27 & 20 & 25 & 22 & 25 \\
\hline Saturated fat & 25 & 20 & 25 & 20 & 25 & 19 & 25 & 22 & 25 \\
\hline MUFA & 30 & 25 & 30 & 24 & 31 & 24 & 29 & 26 & 30 \\
\hline PUFA & 23 & 18 & 24 & 18 & 23 & 17 & 20 & 17 & 22 \\
\hline Thiamin & 22 & 18 & 21 & 18 & 22 & 18 & 22 & 18 & 24 \\
\hline Total Niacin & 39 & 36 & 39 & 36 & 40 & 36 & 39 & 37 & 38 \\
\hline Vitamin B6 & 25 & 22 & 25 & 22 & 26 & 22 & 23 & 21 & 24 \\
\hline Vitamin B12 & 35 & 28 & 34 & 26 & 39 & 28 & 35 & 29 & 33 \\
\hline Vitamin D & 29 & 22 & 31 & 26 & 32 & 24 & 25 & 19 & 22 \\
\hline Iron & 21 & 16 & 21 & 15 & 22 & 15 & 19 & 17 & 19 \\
\hline Zinc & 39 & 32 & 39 & 31 & 41 & 31 & 39 & 34 & 35 \\
\hline Sodium & 29 & 25 & 30 & 26 & 31 & 25 & 28 & 25 & 26 \\
\hline
\end{tabular}

* M: Men, W: Women

The proportions of adults consuming total meat, red meat, processed meat, and 'poultry \& game' were $98,82,82$, and $80 \%$, respectively. Mean intakes of total meat were $163 \mathrm{~g} / \mathrm{d}$ for men (red meat: $66 \mathrm{~g} / \mathrm{d}$, processed meat: $43 \mathrm{~g} / \mathrm{d}$, poultry \& game: $54 \mathrm{~g} / \mathrm{d}$ ) and $101 \mathrm{~g} / \mathrm{d}$ for women (red meat: $38 \mathrm{~g} / \mathrm{d}$, processed meat: $24 \mathrm{~g} / \mathrm{d}$, poultry \& game: $39 \mathrm{~g} / \mathrm{d}$ ). Overall, meat and meat products contributed $17 \%$ of energy intake. Relative to their contribution to energy intake, meat and meat products contributed greater proportions of protein, mono-unsaturated fat, B vitamins, zinc and vitamin D (most age/sex groups), and similar proportions of iron. However, they also contributed greater proportions of fat (total and saturated) and sodium intakes. These findings show that while meat and meat products are a key contributor to intakes of important macro- and micro-nutrients in Irish adults, they also contribute high proportions of total fat, saturated fat and sodium.

The authors acknowledge the contribution of Meat Technology Ireland (MTI), a co-funded industry/Enterprise Ireland Technology Centre funded through the Technology Centre programme (TC 2016 002). The National Adult Nutrition Survey was funded by the Irish Department of Agriculture, Fisheries and Food under the Food for Health Research Initiative (2007-2012).

1. Bender A (1992) FAO Food Nutr Pap 53, 1-91.

2. Food Standards Agency (2002) McCance \& Widdowson's The Composition of Foods. Cambridge: RSC.

3. Black LJ, Ireland J, Møller A et al. (2011) J Food Compost Anal 24(7), 1017-1023. 